\title{
Thanks to the Reviewers of the Korean Journal of Radiology
}

The editors of the Korean Journal of Radiology appreciate the efforts of manuscript reviewers who completed their reviews for the journal from Dec. 2012 to Nov. 2013 to help make the most appropriate decisions on the manuscripts. We sincerely express our gratitude to all the reviewers listed below for their time and expertise. Through their dedicated service to the journal, the current Korean Journal of Radiology has been shaped and is evolving into a multifaceted journal of radiological science and education. Please note that the individuals listed on the 2013 masthead for the Korean Journal of Radiology were not included.

Yeon Hyeon Choe, M.D. Editor-in-Chief

$\begin{array}{lll}\text { Byeong-Cheol Ahn } & \text { Gi Jeong Cheon } & \text { Sang Il Choi } \\ \text { Kookjin Ahn } & \text { Stephen Cheung } & \text { Soo-Jung Choi } \\ \text { Sung Soo Ahn } & \text { Kuan-Rau Chiou } & \text { Yoon Jung Choi } \\ \text { Ozgur Akdemir } & \text { Arthur Cho } & \text { Young Jun Choi } \\ \text { Young-Sil An } & \text { Bum Sang Cho } & \text { Yun Sun Choi } \\ \text { Alberto Avolio } & \text { Eun Suk Cho } & \text { Yun Young Choi } \\ \text { Jae-Ik Bae } & \text { Ihn-Ho Cho } & \text { Semin Chong } \\ \text { Min Sun Bae } & \text { In-Jeong Cho } & \text { Ki Seok Choo } \\ \text { Jung Hwan Baek } & \text { Jae Ho Cho } & \text { Eun Ju Chun } \\ \text { Seung Yon Baek } & \text { Kyu Ran Cho } & \text { Jae-Joon Chung } \\ \text { Seung Kook Baik } & \text { Nariya Cho } & \text { Jin Chung } \\ \text { Hee-Seung Bom } & \text { Yun Ku Cho } & \text { Wook-Jin Chung } \\ \text { Louis-Martin Boucher } & \text { Dongil Choi } & \text { Yong Eun Chung } \\ \text { Marc A. Brockmann } & \text { Eui-Young Choi } & \text { Kyung-Hyun Do } \\ \text { Jae Ho Byun } & \text { Hyuck Jae Choi } & \text { Lianfang Du } \\ \text { Lanciego Carlos } & \text { Hyun Seok Choi } & \text { Hong Eo } \\ \text { Eun Suk Cha } & \text { Ja-Young Choi } & \text { Alessandro Furlan } \\ \text { Jang Gyu Cha } & \text { Ji Soo Choi } & \text { Dong Il Gwon } \\ \text { Joohee Cha } & \text { Jin Woo Choi } & \text { Doo Hoe Ha } \\ \text { Sang-Hoon Cha } & \text { Jin Wook Choi } & \text { Eun Ju Ha } \\ \text { Eun Jin Chae } & \text { Jin-Ho Choi } & \text { Soo-Youn Ham } \\ \text { Jee Won Chai } & \text { Jin-Young Choi } & \text { Dae Hee Han } \\ \text { Il Soo Chang } & \text { Joon-Il Choi } & \text { Heon Han } \\ \text { Jung Min Chang } & \text { Jung-Ah Choi } & \text { You Mie Han } \\ \text { Yun-Woo Chang } & \text { Nami Choi } & \text { Young-Min Han } \\ & & \end{array}$

Mutsumi Hayakawa

Suk Hee Heo

Brian R. Herts

Hye Suk Hong

Hyun Sook Hong

Suk-Joo Hong

Yongmin Huh

Jin Hur

Ji Young Hwang

Jung Hwa Hwang

Sung Ho Hwang

Sung Il Hwang

Seung Hyup Hyun

Soo Ah Im

Geon-Ho Jahng

Kyung Mi Jang

Bo-Kyung Je

Keum Nahn Jee

Tae Joo Jeon

Doo-Shin Jeong

Hwan-Jeong Jeong

Sun Young Jeong

Woo Kyoung Jeong

Yeon Joo Jeong

Kwangnam Jin 
Masahiro Jinzaki

Min Woo Jo

Ijin Joo

Seon Kwan Juhng

Ah Young Jung

Dae Chul Jung

Jung Im Jung

Seung Eun Jung

Sung Il Jung

Bong Joo Kang

Byung Chul Kang

Chae Hoon Kang

Chang Ho Kang

Eun-Ju Kang

Eun-Young Kang

Joon-Won Kang

Keon Wook Kang

Won Jun Kang

Bharti Kataria

Zenichiro Kato

Bohyoung Kim

Byung Moon Kim

Chan Kyo Kim

Dong Hun Kim

Dong Wook Kim

Eun Young Kim

Eung Yeop Kim

Eunhee Kim

Ho Sung Kim

Hye Jung Kim

Hyeonjin Kim

Hyo-Cheol Kim

Hyoung Jung Kim

Hyung-Kwan Kim

In-One Kim

Jae-Hun Kim

Jee Young Kim

Jeong A Kim

Ji Hye Kim

Ji-hoon Kim

Jin Hee Kim

Jin Woong Kim

Jinna Kim

Joo Hee Kim

Jung Hoon Kim

Kwanggi Kim

Kyoung Won Kim
Kyung Rae Kim

Kyung Won Kim

Kyuseok Kim

Mi Jeong Kim

Mi-hyun Kim

Min Ju Kim

Min Jung Kim

Namkug Kim

Ok Hwa Kim

Pyo Nyun Kim

Sam Soo Kim

Sang Youn Kim

Se Hyung Kim

See Hyung Kim

Seong Hyun Kim

Seong-Jang Kim

Seung Ho Kim

Seung Up Kim

So Yeon Kim

Soo-Mee Kim

Sun Ho Kim

Sun Mi Kim

Sung Hun Kim

Sungjun Kim

Tae Hoon Kim

Tae Kyoung Kim

Tae Sung Kim

Yang Min Kim

Yeo Ju Kim

Yoon Kyung Kim

You Me Kim

Young Jun Kim

Young Kon Kim

Yu Kyeong Kim

Shigeru Kiryu

Eun Sook Ko

Eun Young Ko

Heung Kyu Ko

Kyungran Ko

Aravind S. Ravi Kumar

Akira Kurata

Kyu-Sung Kwack

Jin Young Kwak

Jong Won Kwon

Jung Hyeok Kwon

Oki Kwon

Soon Tae Kwon
Ellen De Langhe

Bae Young Lee

Chang Hee Lee

Chang Hyun Lee

Deok Hee Lee

Dong Ho Lee

Edward W. Lee

Eun Sun Lee

Hee Jung Lee

Heon Lee

Ho Yun Lee

Hye-Jeong Lee

In Ho Lee

In Sook Lee

Jae Young Lee

Jae-Hoon Lee

Jee Young Lee

Jeong Hyun Lee

Jeong Kyong Lee

Jeong Won Lee

Ji Won Lee

Jongmee Lee

Jongmin Lee

Joon Woo Lee

Ju Hee Lee

Jung Pyo Lee

Ki Yeol Lee

Kyoung Ho Lee

Mi-Jung Lee

Min Hee Lee

Min Woo Lee

Sang Min Lee

Sang Yong Lee

Sang-Chol Lee

Sang-Woo Lee

Seon-Kyu Lee

Seung Soo Lee

Sheen-Woo Lee

So Yeon Lee

Soon Jin Lee

Su Jin Lee

Sun Joo Lee

Sung Moon Lee

Wen-Jeng Lee

Won Hyeong Lee

Yoon Jin Lee

Young Han Lee
Young Hen Lee

Young Joon Lee

Young Seok Lee

Gye-Yeon Lim

Hyun Kyung Lim

Joon Seok Lim

Soo Mee Lim

Young-Suk Lim

Bin Lu

Lorenzo Mannelli

Yumi Matsushita

Alexander McKinney

Siroos Mirzaei

Hee Jung Moon

Sung Gyu Moon

Sung Kyoung Moon

Dong Gyu Na

So Won $\mathrm{Oh}$

Young Taik Oh

Jin Chul Paeng

Auh Whan Park

Byung Kwan Park

Chang Min Park

Duk-Woo Park

Eun-Ah Park

Hee Jin Park

Hee Sun Park

Jaeseok Park

Ji Kang Park

Ji Seon Park

Kyung Joo Park

Mi Jung Park

Mi-Suk Park

Sang Joon Park

Sung Bin Park

Sung-Hong Park

Sung-Tae Park

Sun-Won Park

Young Mi Park

Jeong Seon Park

Perry J. Pickhardt

Junhee Pyo

Jens Rassweiler

Sung Eun Rha

Hyunchul Rhim

Hong Gee Roh

Chang-Woo Ryu 
Junsun Ryu

Yasemin Sanli

Yozo Sato

Bo Kyoung Seo

Sang Won Seo

Cheong-Il Shin

Hee Jung Shin

Jung Hee Shin

Kyung Min Shin

Myung Jin Shin

Na-Young Shin

Sang Soo Shin
Su-Mi Shin

Sung Wook Shin

Young Ho So

Chul-Ho Sohn

Yu-Mee Sohn

Eun Ju Son

Ho-Taek Song

Sang Hyun Suh

Sang-il Suh

Dong-Wook Sung

Yon Mi Sung

Kunihiko Teraoka
Harriet C. Thoeny

Hyung Jin Won

Je Hwan Won

Ok Hee Woo

Dong Hyun Yang

Ik Yang

Masato Yasuta

Chin A Yi

Hwan Seok Yong

Seung Min Yoo

So-Young Yoo

Chang-Jin Yoon
Dae Young Yoon

Hyun-Ki Yoon

Jeong Hee Yoon

Jung Hyun Yoon

Seong Kuk Yoon

Soon Ho Yoon

Young Cheol Yoon

Ji Hyun Youk

Wei Yu 